\title{
Parenting experiences among fathers of prematurely-born children with cerebral palsy in South Korea
}

\author{
Jisun Park ${ }^{1}$, Kyung-Sook Bang ${ }^{2}$ \\ ${ }^{1}$ Head Nurse, Seoul National University Hospital, Seoul; ${ }^{2}$ Professor, College of Nursing, Research Institute of Nursing Science, Seoul National University, Seoul, Korea
}

Purpose: The symptoms and impairments caused by cerebral palsy usually require long-term treatment, resulting in a substantial burden on the family of affected children. This study explored the experiences of fathers with prematurely-born children with cerebral palsy, with a focus on how such experiences influenced their families. Methods: A qualitative case study method was used. Nine subjects were recruited from April 2018 to June 2019 at one hospital, and each was interviewed three times by a neonatal nurse. Results: Five core experiences of fathers were identified: regret for an insufficient initial response, confronting my child born as a premature baby, the position of being a dad who can't do anything, the process of treatment like a tunnel with no exit, and a father's getting meaning in life through children. These stories covered an individual's timeline and family interactions. Conclusion: Our findings suggest that fathers of prematurely-born children tend to suppress their emotions; therefore, a novel intervention program to encourage fathers' emotional expression and to support healthier interactions with their families is needed. Moreover, our findings could contribute basic information for the construction of a community-based support system to aid families, including prematurely-born children and other persons with impairments.

Key words: Premature infant; Cerebral palsy; Fathers; Parenting; Qualitative research

\section{Corresponding author Kyung-Sook Bang}

https://orcid.org/0000-0001-9902-9716

College of Nursing, Seoul National University,

103 Daehak-ro, Jongno-gu, Seoul 03080, Korea

TEL +82-2-740-8819 FAX +82-2-765-4103

E-MAIL ksbang@snu.ac.kr

*This article was adapted from a dissertation by Jisun Park in partial fulfillment of the requirements for the doctoral degree at Seoul National University. *This study was supported by the research fund by Korean Academic Society of Child Health Nursing in 2019.

Received Dec 19, 2020 Revised Jan 8, 2021 Accepted Jan 12, 2021 (a) This is an Open Access article distributed under the terms of the Creative Commons Attribution NonCommercial License (http://creativecommons.org/licenses/by-nc/4.0/) which permits unrestricted noncommercial use, distribution, and reproduction in any medium, provided the original work is properly cited.

\section{INTRODUCTION}

The proportion of premature infants in South Korea (hereafter, Korea) has increased from $5.7 \%$ in 2009 to $8.1 \%$ in 2019. Moreover, in cases of multiple fetuses, $63.4 \%$ of children were born at under 37 weeks of age in 2019, which was higher than the figure of $52.5 \%$ in 2009 , and low-birth-weight infants (under 2,500 grams) constituted 6.6\% of all births in 2019 [1].

High-risk newborns, including premature and underweight infants, are admitted to the neonatal intensive care unit (NICU) immediately after birth. The artificial and physical environment of the NICU negatively affects the normal sensory and central nervous system development of preterm infants, and can lead to sequelae such as developmental disabilities, growth retardation, and behavioral disorders [2,3].

Throughout the world, with the development of perinatal medicine and neonatal intensive care over the past 30 years, the mortality rate of premature infants has declined rapidly. In Japan, the survival rate is reported to exceed $80 \%$. Despite this high survival rate, the risk of developing neurodevelopmental disorders, mainly cerebral palsy (CP), in extremely low-birth-weight (ELBW) babies is still high, and has been reported to range from $8.2 \%$ to $9.9 \%$, in both Korean and international studies [4-6]. 
The symptoms of CP appear from birth and persist throughout life. Children with $\mathrm{CP}$ experience impaired sensation, communication, and cognition, and have many limitations in performing daily activities such as eating, getting dressed, bathing, and moving [7]. In addition, because children's function is increasingly limited as they grow requiring additional rehabilitation treatment and care, the burden on families increase over time [8,9].

Fathers with infants and toddlers begin to form a sense of paternity through their initial interactions with their children, which continue to influence the father-child relationship thereafter. At the same time, the father exerts an influence on the children's cognition, sociality, and physical development. However, despite this importance, the role of the mother tends to be predominantly emphasized in childhood, and the importance of the father's role is overlooked [10]. However, as women's economic activity has increased and the discourse of paternity has changed due to the shift towards the nuclear family, modern fathers have become more active in raising children and prefer a friendly father image unlike their fathers' generation $[11,12]$.

Despite the fact that fathers have been recognized as important participants in the care of infants in recent years, the experience of fathers with prematurely-born children is not well represented in the Korean literature. Although studies have been conducted on fathers' experiences of raising premature infants in the United States and other countries, little is known about the experience of fathers raising children born prematurely and diagnosed with $\mathrm{CP}$.

The purpose of this study was to gain insights into the life experiences of fathers raising children with $\mathrm{CP}$ who were born as premature infants, and how this affected the lives of their families. The research questions were 1) "What experiences did fathers of premature babies have during hospitalization and treatment in the NICU?" and 2) "What experiences did fathers have while raising children diagnosed with CP after discharge from the NICU, and what did these experiences mean for family members' lives?"

\section{METHODS}

\section{Study Design}

This study used a multi-case study analysis method with qualitative case studies in order to gain insights into the life experiences of fathers raising children with $\mathrm{CP}$ born as premature infants, and how those experiences influenced family life. The reasons for using this method were as follows. First, the participants' experiences of life related to child-rearing were based on the issues that emerged through a series of processes, starting from the birth of a premature infant to the present, when they cared for their children with CP. Second, it was necessary to collect data through various sources such as sufficient in-depth interviews, telephone interviews, field notes, and medical record reviews. In addition, considering the characteristics of the qualitative case study method (intracase analysis and inter-case analysis), it was judged that the application of the qualitative case study method was appropriate in order to obtain an in-depth understanding of fathers' life experiences related to child-rearing.

\section{Participants}

The participants of this study were fathers who experienced the birth of a premature baby and were raising a child diagnosed with $\mathrm{CP}$, selected during outpatient observation of the child. Purposive sampling was used to select participants who could provide in-depth experiences relating to the research problem.

Recruitment documents were posted in the hospital's neonatal outpatient clinic to recruit study participants, and $17 \mathrm{fa}$ thers who met the criteria were introduced by the outpatient coordinator. The study was then specifically explained to the 17 fathers by phone or e-mail, and within a week to a month, 10 fathers consented to participate in the study and made an appointment for an interview. However, one out of the $10 \mathrm{fa}$ thers who participated in the interview was excluded from the study because he complained of a change in emotions during the first interview and decided to stop the interview. Finally, nine fathers participated in the study. The sociodemographic characteristics of the participants are presented in detail in Table 1.

\section{Ethical Considerations and Data Collection}

This study was conducted after obtaining approval from the Institutional Review Board (IRB No. 1709-108-887) of the affiliated university hospital. Detailed information about the study was provided to the participants, who made inquiries about the study by phone or email, and their voluntary consent was confirmed. The study participants were then given a detailed explanation about the purpose of the study, the process of collecting data, the period of participation in the study, the recording of the interview content, and the storage method and time limit of the study data, and they were reassured that the collected data would not be used for any purpose other than the purpose of the study.

Data collection was conducted through two individual in-depth interviews with each participant between April 2018 and June 2019. The location and time of the interviews were 
Table 1. Characteristics of the Participants

\begin{tabular}{|c|c|c|c|c|c|c|c|c|c|c|}
\hline & & Yeon & Jin & Dan & Hwan & Soo & Woon & Min & Joon & Seok \\
\hline \multirow[t]{4}{*}{ Father } & Age (year) & 40 & 38 & 30 & 31 & 37 & 36 & 45 & 37 & 51 \\
\hline & Education & University & University & $\begin{array}{l}\text { High } \\
\text { school }\end{array}$ & $\begin{array}{l}\text { High } \\
\text { school }\end{array}$ & University & $\begin{array}{l}\text { Graduate } \\
\text { school }\end{array}$ & University & University & University \\
\hline & Job & Employee & Employee & $\begin{array}{c}\text { Self- } \\
\text { employment }\end{array}$ & $\begin{array}{l}\text { Daily } \\
\text { work }\end{array}$ & $\begin{array}{c}\text { Self- } \\
\text { employment }\end{array}$ & $\begin{array}{l}\text { Office } \\
\text { worker }\end{array}$ & Employee & Employee & Employee \\
\hline & Socio-economic status & High & High & Medium & Low & High & High & Medium & Medium & Medium \\
\hline \multirow[t]{10}{*}{ Child } & Gestational age (week) & $26+2$ & $25+4$ & $30+4$ & $25+3$ & $27+1$ & $30+2$ & $29+0$ & $24+6$ & $26+5$ \\
\hline & $\begin{array}{l}\text { Body weight at birth } \\
\text { (gm) }\end{array}$ & 1,020 & 900 & 2,020 & 860 & 520 & 1,430 & 930 & 710 & 980 \\
\hline & Sex & Female & $\begin{array}{l}\text { Female } \\
\text { (1st baby } \\
\text { of twins) }\end{array}$ & $\begin{array}{l}\text { Female } \\
\text { (2nd baby } \\
\text { of twins) }\end{array}$ & Male & Male & Male & $\begin{array}{l}\text { Female } \\
\text { (2nd baby } \\
\text { of twins) }\end{array}$ & Male & Male \\
\hline & Corrected age (month) & 32 & 24 & 35 & 19 & 30 & 12 & 20 & 18 & 15 \\
\hline & K-Bayley-III & & & & & & & & & \\
\hline & -Cognitive & $8 \mathrm{~m}$ & $17 \mathrm{~m}$ & $4 \mathrm{~m}$ & $14 \mathrm{~m}$ & $17 \mathrm{~m}$ & $6 \mathrm{~m}$ & $16 \mathrm{~m}$ & $11 \mathrm{~m}$ & - \\
\hline & -Receptive language & $15 \mathrm{~m}$ & $15 \mathrm{~m}$ & $4 \mathrm{~m} \mathrm{10d}$ & $11 \mathrm{~m}$ & $22 \mathrm{~m}$ & $4 \mathrm{~m} \mathrm{10d}$ & $19 \mathrm{~m}$ & $7 \mathrm{~m}$ & - \\
\hline & -Expressive language & $15 \mathrm{~m}$ & $19 \mathrm{~m}$ & $6 \mathrm{~m}$ & $11 \mathrm{~m}$ & $18 \mathrm{~m}$ & $2 \mathrm{~m}$ & $13 \mathrm{~m}$ & $7 \mathrm{~m}$ & - \\
\hline & -Gross motor & $6 \mathrm{~m}$ & $9 \mathrm{~m}$ & $4 \mathrm{~m}$ & $11 \mathrm{~m}$ & $11 \mathrm{~m}$ & $20 \mathrm{~d}$ & $15 \mathrm{~m}$ & $9 \mathrm{~m}$ & - \\
\hline & -Fine motor & $11 \mathrm{~m}$ & $17 \mathrm{~m}$ & $3 \mathrm{~m}$ & $15 \mathrm{~m}$ & $20 \mathrm{~m}$ & $5 \mathrm{~m}$ & $17 \mathrm{~m}$ & $10 \mathrm{~m}$ & - \\
\hline
\end{tabular}

d, day; m, month.

chosen by the participant. The interview times varied from 1 hour and 30 minutes to 3 hours per session depending on the progress of the interview, and the interval between the first and second interviews was 2 to 4 weeks. Subsequently, a 10-minute telephone interview was conducted to confirm the results with the study participants.

The first interview was initiated with the following unstructured questions: "Can you tell me about your memories of conceiving a baby or the experience of preparing to conceive a baby with your wife?", "Can you tell me about your experiences related to the birth of your premature baby?", "Tell me about your experiences while your baby was staying and undergoing treatment at the NICU", "What was the treatment and progress while your child was growing up?", "Tell me about major memorable events and circumstances at that time", "What was the health status of your baby at the time of discharge and what physical, psychological and life changes occurred in your family?", "When did you find out that your child had a developmental delay?", and "Tell me the extent of developmental delay of the child, and your experiences during treatment".

In the second interview, the researcher summarized the content of the first interview, asked if there was a more specific experience, and used the following linking questions about the related experiences: "What was the overall course of the case?" and "What were your thoughts and feelings at that time?" At the end of each interview, the researcher asked how the participant felt about the interview, and expressed gratitude for sharing their feelings with the researcher.

\section{Data Analysis}

In this study, based on the qualitative case study method proposed by Yin [13], data were collected and analyzed simultaneously. The transcribed content was read repeatedly, and concepts were extracted and categorized to derive themes. As the collected data were analyzed, theoretical saturation (the point at which no new categories were found anymore) was confirmed, and then the recruitment of participants and data collection were terminated. In addition, on-site notes were written to record various aspects occurring in the field at each interview. In addition to the main interview details, the study participants' moods, thoughts, coping methods, and case solutions and results were recorded. The K-Bayley-III score was confirmed through the father's memory and then supplemented through a medical record review. The K-Bayley-III score is for infants and toddlers from 16 days to 42 months and 15 days of age, and it consists of a total of five areas: cognitive scale, language scale, motor scale, social-emotional scale and adaptive behavior scale. Among these five areas, the cognitive scale, language scale (receptive language, expressive language), and motor scale (gross motor, fine motor) were used. 
Within a week after the end of each interview, the researcher transcribed the interview content, and it was compared and reviewed repeatedly to prevent omission or misrepresentation of the original data. For data analysis, the researcher described the experiences of individual participants, performed an intra-case analysis of fathers, and then conducted an inter-case analysis. For intra-case analysis, the fathers' experiences were identified and analyzed by setting the individual subjects participating in the study as cases. The researcher intensively analyzed each case while reading the manuscripts repeatedly, and wrote individual memos in order to understand the flow of each case. At the same time, the researcher paid attention to new content that could be discovered when comparing previously examined cases. Based on the intra-case analysis results, the fathers' experiences and the content related to their current child-rearing experiences were integrated into sub-themes, and similar sub-themes were collected and presented as single themes.

\section{Ensuring Trustworthiness}

The four trustworthiness criteria of credibility, dependability, confirmability, and transferability proposed by Guba and Lincoln [14] were considered to ensure that this study presents reliable qualitative research, along with the criteria proposed by Sandelowski for securing the rigor of qualitative research [15].

First, credibility refers to how accurately and faithfully the research results describe and interpret actual experiences. In this study, interviews were conducted using open and semistructured questions in order to increase the reliability of the data. After recording all the interviews, the researcher directly transcribed them within a short time so that there was no omission or distortion of the data. The results of the study were compared with the original statements of the study participants.

Second, dependability corresponds to external validity in quantitative research, in this study, data were collected and analyzed until the statements of the study participants selected by the purpose-built expression method appeared repeatedly at the state of saturation, where new content was no longer produced, and the findings were then confirmed with nurses and outpatient coordinators working in the NICU.

Third, confirmability corresponds to the reliability of quantitative research, and is a criterion for evaluating whether the process of deriving results through data collection and analysis is consistent. To this end, the qualitative case study method suggested by Yin [13] was faithfully reflected and the process of collecting and analyzing data was explained in detail, as well as the process of deriving the study results, so that the process could be clearly understood.

Fourth, transferability refers to the objectivity of quantitative research and involves maintaining neutrality by minimizing research bias in the research process and results. During the course of this study, the researcher took time to consider in advance the assumptions, understanding, and experiences so that the past experiences of nursing premature infants and caregivers in the NICU would not introduce any prejudice.

The first author specialized in child nursing in graduate school and worked in the NICU for 10 years after graduation, and realized that understanding the families of premature infants is important for providing care. While meeting parents for a long time as a nurse, the researcher realized that childrens' progress should be monitored not only during the acute phase in the intensive care unit, but also after discharge given their risk of neurodevelopmental disorders and need to assess their symptoms and prognosis. In addition, the researcher used qualitative nursing research methodology, qualitative data analysis, and family studies qualitative research methods in the doctoral course at graduate school, and continued preparing for qualitative research by attending workshops and special lectures hosted by the Korean Association for Qualitative Research. The researcher has also participated in focus group research several times as a co-author.

\section{RESULTS}

The nine participants' descriptions of the lives of their children and their own experiences as fathers were compiled for each case with consideration of the passage of time and interactions in each family. In this study, only an inter-case analysis is presented, focusing on participants' common life experiences. Regardless of whether the experiences were common or individual, we explored the meanings of participants' experiences, looking at what the events meant and how they affected the participants' lives. It was also found that those experiences were not limited to the fathers who participated in this study alone, but could be shared with other fathers who experienced the birth of a premature baby and were raising children with neurological sequelae. With a focus on the main experiences of the nine fathers, what the fathers wanted to say, examples, and the meanings of their experiences were organized into five themes and 14 sub-themes. The names for description in this study are alias (Table 2).

\section{Regret for an Insufficient Initial Response}

The interviews with the fathers involved in the study began with recalling the memories of all of their experiences during their wives' pregnancies. In most cases of premature infants 
Table 2. Themes and Sub-themes of Parenting Experiences of the Fathers of Prematurely-born Children with Cerebral Palsy

\begin{tabular}{|c|c|}
\hline Themes & Sub-themes \\
\hline Regret for an insufficient initial response & $\begin{array}{l}\text { Resentment towards the response of medical staff that did not meet expectations } \\
\text { Sadness for the wife's inability to perform adequate self-care during pregnancy } \\
\text { Sadness for not being able to help their wives }\end{array}$ \\
\hline Confronting my child born as a premature baby & $\begin{array}{l}\text { Surprise and regret for the appearance of a child who was so small and fragile } \\
\text {. Differences in perspectives between fathers and others' view of premature infants }\end{array}$ \\
\hline The position of being a dad who can't do anything & $\begin{array}{l}\text { Concern about the guilt and depression of the child's mother } \\
\text { - Dad's emotional difficulties that cannot be revealed } \\
\text { A feeling of helplessness due to the growing conflict between partners }\end{array}$ \\
\hline The process of treatment like a tunnel with no exit & $\begin{array}{l}\text { Facing the hard moments of life } \\
\text { Rehabilitation, the most important treatment that can be given to my child } \\
\text { Relief due to the child's developmental improvement, and desire for more } \\
\text { improvement in the future }\end{array}$ \\
\hline A father getting meaning in life through children & $\begin{array}{l}\text { The financial burden faced by dad } \\
\text { - My child who became the reason for my existence } \\
\text { Setting goals for my life with my child }\end{array}$ \\
\hline
\end{tabular}

admitted to the NICU, there had been high-risk pregnancy and delivery due to problems with the mother or child. The medical response was thought to be insufficient or inadequate, so that fathers were disappointed.

\section{1) Resentment towards the response of medical staff that did not meet expectations}

Fathers faced the moment of birth of a premature baby through unexpected, sudden pain experienced by their wives, or signs of premature birth found during regular hospital examinations. Contrary to one father's expectation that a quick visit to the obstetrics and gynecology department would solve the problem, the medical staff he met at the hospital did not help him.

My wife had a stomachache, so she went to the obstetrics and gynecology department of the nearby hospital. However, they did not even look at the ultrasound, and wasted time. However, since she continued bleeding, the doctor recommended going to a university hospital after seeing an ultrasound. It was too late, and suddenly, a doctor called me, and the baby's head was already showing. There was no choice but premature birth. (Joon's father)

\section{2) Sadness for the wife's inability to perform adequate self-care during pregnancy}

In addition to the poor initial response at the hospital and resentment against the medical staff, one of the other reasons that eventually led to the birth of a premature infant was insufficient maternal self-care, and husbands expressed regret for their wives.

My wife took a break from work for a while and tried artificial fertility for three months, and after success, she returned to work. She had to stay late at night, so I think it's because my wife was under a lot of stress. (Jin's father)

\section{3) Sadness for not being able to help their wives}

In addition to their wives' health care during pregnancy, there were several other reasons for giving birth prematurely. These factors (e.g., health status or the residential environment) did not necessarily reflect anyone's active choice, but the fathers thought that it was a pity that they could not help their pregnant wives.

Joon's mother is very weak. When she was sick, she weighed a little over 30 and only skin and bones. Originally, she had a periodic vomiting syndrome of unknown cause and was vomiting a lot during pregnancy. I think the child came down because of that pressure. (Joon's father)

The house we live in was very small, and at that time, the people next door was remodeling their home. Hwan's mother, who was alone at home, couldn't get enough rest because of the construction and was also quite stressed. (Hwan's father)

\section{Confronting My Child Born as a Premature Baby}

1) Surprise and regret for the appearance of a child who was so 


\section{mall and fragile}

Fathers went beyond their regret regarding the poor initial response, and were faced with the need to respond to the early birth of a child. Regardless of whether the pregnancy had been long awaited or was a sudden pregnancy before marriage, the fathers met their children very suddenly, and were surprised by the appearance of a child who was so small and fragile. They expressed sadness and regret.

The baby was born, but it looked like it was still in danger in the incubator. He could not breathe well, they had plugged in a venous line, and he was breathing with a ventilator. I felt like my heart was being ripped apart. I cried for the first time then. (Hwan's father)

\section{2) Differences in perspectives between fathers and others' views of premature infants}

While starting and experiencing the treatment process for their premature infants, fathers often heard the reason "because of being a premature baby" to explain various difficulties arising during the treatment process. Unlike the partners of premature babies, medical staff and others expressed different perspectives on premature infants.

No matter how premature he is, I have never thought of it as a bad situation. It seemed to work out. I think I had that kind of faith. But every time I heard that I had to be prepared for his end, I felt frustrated. The kid is growing well, and it's all miracles right now. I hated everyone near me, who talked of negative results. (Hwan's father)

Everyone said that Seok was doing so poorly because he had been born as a premature baby, but of course I knew that. No matter how difficult the case is, there will be no answer if you look at the issue through the frame of a premature baby. (Seok's father)

\section{The Position of Being a Dad Who Can't Do Anything}

Through the experiences of the fathers, it was possible to analyze the various psychological states that they experienced, as well as their position and role of the fathers; overall, their circumstances could be expressed as the position of a father who was unable to function at all. In light of their concerns about the mothers' guilt and depressed mental health, the fathers' current state of mind was bound to be concealed. Fathers also felt helpless about the conflict with their wives due to the problems faced by their children.

1) Concern about the guilt and depression of the child's mother Fathers feared that bad things would happen to their wives because they had severe feelings of guilt and depression related to the birth of a premature baby.

I was having a lot of trouble mentally, but it may be harder for my wife than me. I worried about my wife's emotions, and I felt like I should not be left alone. I did not go to a psychiatric department for treatment, but I cried more often and felt a loss of confidence compared to before, so I do not think I felt comfortable then either. (Jin's father)

\section{2) Dad's emotional difficulties that cannot be revealed}

Although fathers played an important role in helping to overcome and support their wives, who were experiencing guilt and depressed feelings, they felt that could not tell anyone about their own emotional state. Sometimes they complained of the difficulty of controlling their emotions, and were just hoping that this situation would pass.

Honestly, I worked outside the house and returned home late, but since I came home, I had to do something. It is also difficult, and I and my wife do not understand each other well. Because they are both new to me, there are such situations. Living is not difficult, but psychologically things are difficult for me. (Dan's father)

When Joon was born and in the hospital, I had to work and was seriously distressed. I was so sorry that Joon's illness was attributable to our fault. I thought about why this happened to me, and it was very hard in terms of economic difficulties. (Joon's father)

\section{3) A feeling of helplessness due to the growing conflict between partners}

At the beginning of the marriage, the fathers expressed that they had happy marriages and respected each other. However, after giving birth to a premature baby and raising a child with a disability, they felt a sense of helplessness that was difficult to resolve, and there was a visible or invisible conflict between husband and wife. However, the fathers simply accepted the current state of conflict as inevitable.

Actually, the structures of thinking of men and women are different, and women think in a more complex way. So it is difficult to adapt to the wife more and more as time passes. (Yeon's father)

Along similar lines, one father expressed regret for the lack of systematic and active support to help his relationship with his wife who had a premature baby. He mentioned that such support is essential. 


\section{The Process of Treatment Like a Tunnel with No Exit}

\section{1) Facing the hard moments of life}

Fathers came to realize that their babies needed rehabilitation, starting with treatment in the NICU and continuing afterwards. Looking at the symptoms of the baby, various injectable drugs, and the sudden surgery and rehabilitation treatment for the baby's survival, they also felt that they were facing difficulties.

Seizures frequently occur when getting a tooth or having a cold. At first, it was scary, rather than surprising. Yeon was also very hard. But now, I am worrying about whether the seizure duration or pattern will get worse. (Yeon's father)

Just one month after the baby was discharged from the hospital, he had cardiac arrest. The doctor told me that he had aspiration pneumonia when feeding a special formula. We did not know that he could get sick like that. I called 911 first, and after I performed CPR about five times, 911 arrived. (Soo's father)

\section{2) Rehabilitation, the most important treatment that can be giv- en to my child}

Fathers all agreed that rehabilitation was the most important treatment for their children and that it was highly necessary. Therefore, after the start of rehabilitation, the infants had been receiving treatment almost every day, except on weekends. They thought that if they took a day off from rehabilitation, the child's condition would return to its original state, and they worried that the child might not be able to walk later.

I have to go to rehabilitation training unconditionally. Among the extremely low birth weight children who did not receive rehabilitation, they could not do anything on their own later. In contrast, some children who received rehabilitation therapy could stand and walk around, and they began to live their daily lives. Yeoni has been to a rehabilitation hospital since she was very young. (Yeon's father)

3) Relief due to the child's developmental improvement, and desire for more improvement in the future

Fathers were already accommodating the developmental delay as if it was a familiar term. However, fathers were relieved when their children experienced developmental improvements, and changed little by little, although the progress was slowly achieved. Since they had the goal of wanting the child to grow up and have the ability to walk and become self-reliant, they were able to continue rehabilitation treatment and feel positive while waiting.

After undergoing rehabilitation for six months, we heard "Soo could be able to walk. Soo definitely got good parents. Let's do something more." (Soo's father)

\section{A Father's Getting Meaning in Life through Children}

\section{1) The financial burden faced by dad}

It was inevitable that fathers worried about medical expenses for their children. Since most families need more than one adult to take care of their children, the mother played the main role, and it was practically impossible to have two earners. Even in a double-income family, one of them took on the role of the main caretaker who cared for the child after a leave of absence, and the other (mostly the father) had the responsibility of paying for treatment.

Hospital expenses cost a lot, and there are a lot of extra expenses as well. Additionally, the economic burden is passed on to my household and we need the money to take care of the eldest child. That is very burdensome for me and my wife. (Soo's father)

Eventually, money is key. Economic conditions control a lot. As time goes by, I think that the situation may be getting worse and worse. (Yeon's father)

\section{2) My child who became the reason for my existence}

For fathers, their children were the reason for their existence, beyond just beings that needed care, children were reasons for the fathers to live. Life was supported by children, and life without children was unthinkable.

We, as a couple, are most grateful for not giving up in this situation, and I hope that our luck gave us strength. The most difficult thing in my life is the baby. For those long years, half of his life, he lived in a hospital. He is always on my mind. (Woon's father)

\section{3) Setting goals for my life with my child}

Fathers' lives are always planned with their children. Their children are always at the center of their lives since their wives become pregnant, and this continues to be true even in difficult circumstances during children's treatment. The fathers expressed the goal of living with their children; while they were confident that the child would get better, they still prepared for the possibility that the situation would not improve.

On the premise that Yeon cannot have a normal life, I 
think so far in advance. I think about spending on a special school or something like that. Wouldn't it be better to spend on a better school, in a better environment, and have more of that than a cramped place? I am thinking about Jeju Island where we can live close to nature. I think it will get better, but it could get worse. (Yeon's father)

\section{DISCUSSION}

The qualitative case study method was used to conduct a multiple case study analysis of the life experiences of fathers raising children with $\mathrm{CP}$ who were born as premature infants. The first emotion that the fathers encountered when they experienced the birth of a premature baby was regret for the insufficient initial response. The fathers thought that the first hospital visit due to the unexpected and sudden signs of premature birth was not very helpful for the mother and child in that situation. This aligns with the findings of previous studies that the fathers of premature infants experience a loss of control and ambivalence during the birth process of their children, but find it difficult to express their feelings and experience a sense of helplessness because of the feeling that there is nothing that they can do for the child being born [16,17]. Since premature birth is a situation where the child must be completely entrusted to the medical staff, they had no choice but to trust the medical staff to look after the baby [18].

In this study, fathers did not expect their child to be admitted to the NICU, and therefore encountered the early birth of a child without information or experience about the NICU. Whether the child was born through a difficult pregnancy process or was conceived by a sudden pregnancy before marriage, fathers were surprised by the sudden arrival of the baby before the end of the 40-week pregnancy period. Parents with newborn babies hospitalized in the NICU were found to experience high levels of stress when they saw their children being treated with various monitors and machines, including the ventilator [19].

The fathers were concerned that their premature babies were too small to be touched, and were not sure whether the children would survive immediately after birth. The fathers had to accept the fact that they were being separated from their babies by hospitalization [20]. These results are similar to the results of previous studies that found that fathers felt like outsiders because they were separated from their children who were born prematurely, and fathers felt like they were observers during their infants' stay in the NICU [21]. In particular, the fathers of high-risk newborns have feelings of loss of control, anxiety, helplessness, and fear of prognosis during the hospitalization period of their children. Medical staff mostly provide interventions that focus primarily on mothers and infants, and fathers feel alienated at this time [22].

Parents of preterm infants have higher levels of stress than parents of full-term infants [23]. Despite negative effects on the experiences and perceptions of parents with children [24], the health status of the child and the experience immediately after birth have a significant impact on the formation of attachment and perceptions of the early father-and-child relationship. Therefore, medical staff should provide accurate information about the health status of premature infants and to give fathers opportunities to meet their children in person and participate in caring so that premature infants and fathers can form a positive attachment to each other [25].

As the paradigm of nursing in the NICU has recently shifted from patient-centered to family-centered nursing, several international hospitals have created an environment in which parents can visit autonomously and they encourage parents' visits. It has been confirmed that as the number of visits and caring activities of parents increases, the stress of parents decreases [26]. In Korea, medical staff should make efforts to prepare active interventions such as self-visiting and to provide family-centered care.

According to the experiences described by the fathers, various psychological states could be examined. One of these states could be expressed as being in the position of a father who seemed to be unable to function at all. Based on the fathers' reported experiences, the mothers of their children felt guilt and depression. In fact, mothers of premature infants hospitalized in the NICU were found to regret and resent the situation in which they had no choice but to give birth prematurely, blaming everything on themselves. At the same time, they experienced a strong urge to give up their children and a fear of why this might happen to them, and the degree of depression felt by mothers was higher when the child born prematurely had a disability $[18,27]$.

In this situation, the husband was the person who was the greatest comfort to the wife. The fathers faced a major burden, but did not express themselves even in difficult situations, and instead just comforted themselves and had to make decisions without being biased by emotion [18]. In the Confucian patriarchal system, men value authority and strictness, and do not show their feelings well [28]. However, in modern society, gender roles have changed, and the father is expected to participate in parenting, aiming to be a father who is like a friend [28].

In addition, in a study on the adaptation process after the discharge of premature infants, the lack of external support was identified as a major condition making it difficult to adapt [29]. It is necessary to provide more accurate and specific information about the child's condition, various medical in- 
formation required for the child, and parenting methods to parents of premature babies. In addition, it is necessary to establish a child rearing support program for parents raising children diagnosed with disabilities, a support system in the community, and a counseling center.

The fathers in the present study stated that they were going through a process of treatment that they felt was like a tunnel with no end. The premature children were admitted to the NICU and had to overcome life-threatening hurdles several times, and but emergency situations could occur after the children were discharged and cared for at home. After the diagnosis of $\mathrm{CP}$, time passed in the course of treatment, including extensive rehabilitation. The moments of life the baby went through were the same as in the life of the father.

The task remaining for fathers who have already overcome the long treatment process is rehabilitation for their children. In addition, since children with $\mathrm{CP}$ have more demands than normal children and require long-term care, the mother, as the main caregiver, may experience psychosocial problems such as depression, anxiety, and burnout [7]. Therefore, constructing a program that reduces depression and parenting stress and promotes the parental role is required.

Although it is very slow, it is a joyful experience for the father to watch a child accomplish each stage of development one by one, overcoming difficult moments of crisis and showing changes. Therefore, fathers can never give up during the rehabilitation process, and they work especially hard. This result is similar to that of previous research according to which it felt like nothing was visible because they were currently in a dark and long tunnel, but the point in the distant future where they can exit the tunnel at the end and go out to a brighter place indicates the end of rehabilitation treatment [27]. Fathers want sufficient information for children in need of continuing rehabilitation treatment. National initiatives and services for families of premature babies should be considered [30].

Nonetheless, a previous study showed that fathers came to face reality, accept the child as family, and think about the future [21]. Likewise, the fathers who participated in this study also derived meaning in life from the existence of children. For these fathers, their children were the reason for their existence and the reason for them to live.

The results of this study are not limited to children with CP, but can be extended to the experiences of fathers raising children with developmental delays due to a broader group of brain lesion disorders or other causes. In terms of nursing practice, the results of this study are expected to contribute to expanding awareness of the lives of children born prematurely and diagnosed with $\mathrm{CP}$, their fathers, and their families, and to help nurses provide the type of nursing care that is actually needed.

\section{CONCLUSION}

This study used the multi-case study analysis method with nine study participants to obtain insights into the life experiences of fathers raising prematurely-born children with $\mathrm{CP}$, and how their family life was affected. Qualitative case study research was conducted. Through the process of interviews with participating fathers, 'the five main experiences of the meaning of life created through children were "regret for an insufficient initial response", "confronting my child born as a premature baby", "the position of being a dad who can't do anything", "the process of treatment like a tunnel with no exit", and "a father's getting meaning in life through children".

The results of this study show that there is a need to develop a differentiated intervention program that supports and helps fathers to more actively express their emotions, relieve stress, and have more positive adaptation in the child-raising process. In addition, it can be used as basic data for establishing a support system and counseling center in the community as well as a parenting support program for parents of children with disabilities.

A limitation of this study is that the age of the children of the participating fathers was 12 months to 35 months, so broader variety of cases that would enable exploration of different experiences according to age was not included. In this study, the experiences of fathers of prematurely-born children with cerebral palsy were examined, but it will be helpful to conduct further research among fathers of premature infants with various diseases and disorders. In future studies, it will be necessary to study the experiences of fathers of premature babies, including more diverse ages and diseases. Fathers' interest and participation in child-rearing are increasing, and in a social atmosphere whether this is considered natural, this study targeted fathers of premature infants, who have not yet been studied in Korea, to obtain a deeper understanding of fathers in clinical settings and local communities in the future.

\section{Conflict of interest}

No existing or potential conflict of interest relevant to this article was reported.

\section{Data availability}

Please contact the corresponding author for data availability.

\section{REFERENCES}

1. Statistics Korea. Birth statistics in 2019 [Internet]. Daejeon: Statistics Korea; 2020 [Cited 2020 December 19]. Available from: 
http://kostat.go.kr/portal/korea/kor_nw/1/2/1/index.board?b mode $=$ read $\& b S e q=\& a S e q=384631 \&$ pageNo $=2 \&$ row $N u m=10 \&$ na vCount $=10 \&$ currPg=\&searchInfo=\&sTarget=title\&sTxt $=$

2. Gorzilio DM, Garrido E, Gaspardo CM, Martinez FE, Linhares MBM. Neurobehavioral development prior to term-age of preterm infants and acute stressful events during neonatal hospitalization. Early Human Development. 2015;91(12):769-775.

https://doi.org/10.1016/j.earlhumdev.2015.09.003

3. Perez-Pereira M, Fernandez P, Gómez-Taibo M, Gonzalez L, Trisac JL, Casares J, et al. Neurobehavioral development of preterm and full term children: Biomedical and environmental influences. Early Human Development. 2013;89(6):401-409.

https://doi.org/10.1016/j.earlhumdev.2012.12.008

4. Kim S, Song IG, Kim YJ, Kim YJ, Shin SH, Lee SH, et al. Outpatient follow-up status and neurodevelopmental outcomes of extremely low birth weight infants. Journal of the Korean Society of Neonatology. 2012;19(1):17-25.

https://doi.org/10.5385/jksn.2012.19.1.17

5. Mikkola K, Ritari N, Tommiska V, Salokorpi T, Lehtonen L, Tammela $\mathrm{O}$, et al. Neurodevelopmental outcome at 5 years of age of a national cohort of extremely low birth weight infants who were born in 1996-1997. Pediatrics. 2005;116(6):1391-1400.

https://doi.org/10.1542/peds.2005-0171

6. Wilson-Costello D, Friedman H, Minich N, Fanaroff AA, Hack M. Improved survival rates with increased neurodevelopmental disability for extremely low birth weight infants in the 1990s. Pediatrics. 2005;115(4):997-1003.

https://doi.org/10.1542/peds.2004-0221

7. Basaran A, Karadavut KI, Uneri SO, Balbaloglu O, Atasoy N. The effect of having children with cerebral palsy on quality of life, burn-out, depression and anxiety scores: A comparative study. European Journal of Physical and Rehabilitation Medicine. 2013; 49(6):815-822.

8. Cheshire A, Barlow JH, Powell LA. The psychosocial wellbeing of parents of children with cerebral palsy: A comparison study. Disability and Rehabilitation. 2010;32(20):1673-1677.

https://doi.org/10.3109/09638281003649920

9. Unsal-Delialioglu S, Kaya K, Ozel S, Gorgulu G. Depression in mothers of children with cerebral palsy and related factors in Turkey: A controlled study. International Journal of Rehabilitation Research. 2009;32(3):199-204.

https://doi.org/10.1097/MRR.0b013e32832607b6

10. Kim JW, Kim YJ. Analysis of trends in research on Korean fathers with young children published in journals between 1980 and 2006. Family and Environment Research. 2007;45(5):25-38.

11. Yang S. Men's fathering experiences focused on tensions and conflict of multiple roles. Journal of Digital Convergence. 2013;11 (2):375-383. https:// doi.org/10.14400/JDPM.2013.11.2.375

12. Han A. The perceptions on the fatherhood of fathers with young children: Q-methodological approach [master's thesis]. Busan:
Pusan National University; 2015. p. 1-109.

13. Yin RK. Case study research: Design and methods. 5th ed. Los Angeles: Sage publications; 2013.

14. Guba EG, Lincoln YS. Effective evaluation: Improving the usefulness of evaluation results through responsive and naturalistic approaches. San Francisco: Jossey-Bass; 1981.

15. Sandelowski M. The problem of rigor in qualitative research. Advances in Nursing Science. 1986;8(3):27-37.

https://doi.org/10.1097/00012272-198604000-00005

16. Park JE, Lee BS. Experience of becoming a father of a high risk premature infant. Journal of Korean Academy of Nursing. 2017;47(2): 277-288. https://doi.org/10.4040/jkan.2017.47.2.277

17. Hugill K, Letherby G, Reid T, Lavender T. Experiences of fathers shortly after the birth of their preterm infants. Journal of Obstetric, Gynecologic, and Neonatal Nursing. 2013;42(6):655-663.

https://doi.org/10.1111/1552-6909.12256

18. Choi E, Lee Y. A mother's experience of hospitalization of her newborn in the neonatal intensive care unit. Child Health Nursing Research. 2018;24(4):407-419.

https://doi.org/10.4094/chnr.2018.24.4.407

19. Kim TI. A study on the perceived stress level of mothers in the neonatal intensive care unit patients. Child Health Nursing Research. 2000;6(2):224-239.

20. Feeley N, Waitzer E, Sherrard K, Boisvert L, Zelkowitz P. Fathers' perceptions of the barriers and facilitators to their involvement with their newborn hospitalised in the neonatal intensive care unit. Journal of Clinical Nursing. 2013;22(3-4):521-530. https://doi.org/10.1111/j.1365-2702.2012.04231.x

21. Lundqvist $P$, Westas LH, Hallström I. From distance toward proximity: Fathers lived experience of caring for their preterm infants. Journal of Pediatric Nursing. 2007;22(6):490-497.

https://doi.org/10.1016/j.pedn.2007.04.008

22. Hollywood M, Hollywood E. The lived experiences of fathers of a premature baby on a neonatal intensive care unit. Journal of Neonatal Nursing. 2011;17(1):32-40.

https://doi.org/10.1016/j.jnn.2010.07.015

23. Howe TH, Sheu CF, Wang TN, Hsu YW. Parenting stress in families with very low birth weight preterm infants in early infancy. Research in Developmental Disabilities. 2014;35(7):1748-1756. https://doi.org/10.1016/j.ridd.2014.02.015

24. Tooten A, Hoffenkamp HN, Hall RA, Braeken J, Vingerhoets AJ, Bakel HJ. Parental perceptions and experiences after childbirth: A comparison between mothers and fathers of term and preterm infants. Birth. 2013;40(3):164-171. https://doi.org/10.1111/birt.12052

25. Kim ES, Cho YA. The effect of fathers' kangaroo care experience of preterm babies on paternal attachment. Journal of Korean Critical Care Nursing. 2017;10(2):45-55.

26. Lee SJ, Choi EK, Park J, Kim HS. Correlations between a flexible parental visiting environment and parental stress in neonatal intensive care units. Child Health Nursing Research. 2019;25(4):377- 
387. https://doi.org/10.4094/chnr.2019.25.4.377

27. Kang HJ. Experiences of mothers of premature infants receiving rehabilitation therapy. Child Health Nursing Research. 2018;24(3): 298-309. https://doi.org/10.4094/chnr.2018.24.3.298

28. Kim M. New types of masculinity represented in TV and its limitations: Focusing on weekend variety programs. Journal of the Korea Contents Association. 2014;4(1):88-96.

https://doi.org/10.5392/JKCA.2014.14.01.088
29. Ji ES, Byun JL, Park HW, Kim MH. Study on the effects of the family support in the very low birth weight infants follow-up: Focus on Dodam Dodam bring-up center. Korean Journal of Perinatology. 2014;25(2):75-82.

https://doi.org/10.14734/kjp.2014.25.2.75

30. Chang YS. Future of neonatology in Korea: The way forward. Journal of the Korean Medical Association. 2016;59(7):506-513. 